\title{
APLICACIÓN DE INSTRUMENTOS LÚDICOS PARA EL DESARROLLO DE LA MOTRICIDAD FINA DE NIÑOS $Y$ NIÑAS DE 4 AÑOS DE EDAD CON VARIABLES DE GÉNERO, CLASE, ETNICIDAD Y TERRITORIAL, INCLUYE USO DE TIC'S
}

Isabel Almeida Reyes, Edison Cando Vaca, Edwin Panchi

Universidad Central del Ecuador

Recibido: 10 - octubre - 2015, aprobado: 28 - octubre - 2015

\section{Resumen}

La fundamentación teórica que sustentó el estudio, se basa en el análisis de la importancia de los primeros 5 años de vida para el desarrollo de capacidades innatas del individuo y la oportunidad que le ofrecen las experiencias significativas. Se partió de un diagnóstico a través de instrumentos de investigación dirigidos a niños, niñas, educadores-as, y padres de familia. La confiabilidad de los instrumentos se determinó por el cálculo del coeficiente Alpha-Crombach. Los datos obtenidos fueron procesados estadísticamente, su análisis permitió redactar las conclusiones del estudio. La importancia de este trabajo radica en la elaboración de un artículo científico y una publicación a ser socializada con las educadoras que contribuyeron con esta investigación, con los-las docentes y estudiantes de la carrera de Educación Parvularia de la Universidad Central del Ecuador y docentes de otras IES.

Palabras clave: niños, motricidad fina, instrumentos lúdicos.

\begin{abstract}
The theoretical foundation that supported this study is based on the analysis of the importance of the first five years of life for the development of individual's innate abilities and the opportunity that the significant experiences offer. It began with a diagnosis through research instruments aimed at children, teachers, and parents. The instruments' reliability was determined by calculating the Alpha-Crombach coefficient. The data obtained were processed statistically, their analysis allowed to draft the study's conclusions. The importance of this work lies on the development of a scientific paper and a publication meant to be socialized with the educators who contributed with this research, with the teachers and students of the School of Early Childhood Education of the Central University of Ecuador and teachers from other IES.
\end{abstract}

Keywords: children, fine motor skills, leisure instruments. 


\section{Introducción}

El proyecto de investigación "Aplicación de los instrumentos lúdicos para el desarrollo de la motricidad fina con variables de género, etnia, clase y territorialidad de la Facultad de Filosofía, Letras y Ciencias de la Educación, de la Universidad Central del Ecuador, dio inicio en abril del año 2013, con la sustentación de un marco teórico, enfocado en los actuales postulados y concepciones del niño y niña concebido como un ciudadano con derechos, aun desde antes del nacimiento.

Esta investigación, la primera en su género realizada en el país, ha puesto en juego la iniciativa, creatividad y sobre todo la experiencia de los docentes investigadores, que se han propuesto en este proceso investigativo, confirmar la capacidad innata de los infantes entre 4 y 5 años y proponer una metodología que responda a sus intereses y necesidades a través de una experiencia concreta que dé paso a su curiosidad, al juego, a la creatividad a partir del desarrollo psicomotor.

Una oportunidad para crecer como profesionales y un paréntesis en el cumplimiento de la cátedra, para responder a la necesidad de la investigación en el nivel inicial y contar con aportes significativos de un entorno social multiétnico, poco conocido y por tanto poco valorado. Un mundo maravilloso de niños y niñas a descubrirse en este proceso de investigación, diferentes etnias y nacionalidades, asentados en el territorio.

El contacto con estos grupos humanos de la Costa, Sierra, Amazonía y Región Insular, a los que se ha llegado con los instrumentos de investigación para determinar el grado de madurez de la "motricidad fina" objeto de este trabajo, no solo arroja hallazgos interesantes y muy particulares; descubre la multiculturalidad del territorio y la riqueza del ser humano en estas tempranas edades donde la inocencia, lucidez y transparencia dejan conocimiento y mensajes de vida, plasmados en este trabajo y en los espíritus de quienes nos sentimos afortunados de ser parte de este proceso de investigación.

En este camino, se han incorporado estudiantes de la carrera de Educación Parvularia en calidad de pasantes y colaboradores, como "encuestadoras y encuestadores" comprometidos con la universidad y con el proyecto, reconociendo que, sin su ayuda desinteresada, no habría sido posible acercarnos a tan grande población, 1963 niños y niñas.

Un camino recorrido para cumplir los objetivos a partir de la planificación de las actividades en sus diferentes fases, la construcción de los instrumentos de investigación, la puesta en práctica en un pilotaje con una población de 615 infantes de cuatro y cinco años, la validación de los instrumentos de investigación y su aplicación a la población.

Este proceso de investigación fue una oportunidad para la creatividad y mejora continua, una puesta en práctica de la experiencia para construir y reconstruir no solo los instrumentos de investigación, sino también los "instrumentos lúdicos" con visión de contextos, para su aplicación.

El equipo de docentes investigadores tuvo el desafío de crear una atmósfera sinergial comprometida con el desarrollo de las fases del proyecto, para el cum- 
plimiento de cada objetivo, generando un valor agregado a los procesos de investigación sobre aplicación de instrumentos lúdicos a partir del conocimiento de los nuevos paradigmas en la educación inicial, comprendiendo al niño y niña, en el aquí y el ahora, generando bienestar que dé sentido a la existencia, a partir de experiencias y vivencias concretas así como proponer métodos que permitan el desarrollo de la motricidad fina de los niños y niñas.

\section{Contenido de la investigación}

Una experiencia única, para docentes y estudiantes involucrados directamente en este proyecto que, a más de cumplir el objetivo planteado, han puesto en evidencia espíritus sensibles que, al contacto con realidades tan nuestras y al mismo tiempo tan lejanas, conmueven y comprometen.

Las políticas públicas están encaminadas al desarrollo integral en la primera infancia, considerando que son los primeros años de vida de una persona los que condicionan su futuro.

La academia ha considerado relevante entre sus tareas la investigación científica. Así, el proyecto en mención, es una respuesta a los afanes de contribuir a la educación de la primera infancia, a partir de este estudio.

Por lo que, el propósito de esta investigación fue diagnosticar el desarrollo de la motricidad fina de niños y niñas de 4 a 5 años de edad con la aplicación de los instrumentos lúdicos, considerando las variables de género, clase, etnicidad y territorial, válidas en un país multicultural y multiétnico como es Ecuador.

Un tema propuesto por primera vez, en la Universidad Central del Ecuador, Facultad de Filosofía, Letras y Ciencias de la Educación y concretamente de la carrera de Educación Parvularia.

Conscientes que una de las tareas de los docentes universitarios, es investigar y en concordancia con el Plan Nacional para el Buen Vivir, objetivo 2, que propone "fortalecer la educación superior con visión científica y humanista"; es satisfactorio compartir una experiencia única en este proceso de investigación sobre la aplicación de instrumentos lúdicos y el desarrollo de la motricidad fina en niños de 4 y 5 años, partiendo del hecho de comprender la importancia de esta área de desarrollo en las nuevas concepciones del niño y niña.

Los fundamentos de este trabajo investigativo, parten de concepciones básicas como:

\section{¿Qué es la lúdica?}

Un proceso ligado al desarrollo humano; es una actitud, una predisposición del ser frente a la cotidianidad, "una forma de estar en la vida", relacionarse con ella en un ambiente de disfrute, goce, felicidad y distensión que produce el juego, sobre todo en los niños y niñas.

La lúdica, fomenta el desarrollo psico-social, la adquisición de saberes, la conformación de la personalidad, encerrando una amplia gama de actividades donde interactúan el placer, el gozo, la creatividad y el conocimiento. 
El juego, concebido desde la antigüedad, como una de las formas de la actividad humana, es parte de la vida de los niños y las niñas sobre todo de los más pequeños del nivel inicial; es la vía fundamental para el desarrollo integral desde lo biológico, cognitivo, psicológico, motriz y social. Razón por demás suficiente para proponer el empleo de los instrumentos lúdicos para el desarrollo de la motricidad fina, comprendiendo que mediante el juego se despierta al conocimiento, adaptación, orientación y transformación del medio que les rodea.

El carácter educativo de la lúdica, pone de manifiesto el desarrollo, descanso y la diversión; aporta a la formación de la personalidad, genera condiciones para adquirir y afianzar conocimientos, determinan el desarrollo de los procesos mentales, el lenguaje y la comunicación; además, se constituye en la estimulación adecuada y oportuna para despertar la creatividad.

La lúdica por tanto, es una estrategia completa para el desarrollo integral del niño y la niña. Para el objetivo de esta investigación, la acción y la manipulación del material concreto, conducen al desarrollo de la motricidad fina en un ambiente de juego y diversión, así confirman los resultados de este trabajo investigativo y desde luego los aportes de investigadores de todos los tiempos.

Para Vigostky "la actividad mental que realiza el niño y la niña al jugar con el objeto, es la característica fundamental que distingue exclusivamente al hombre como ser humano" (p. 34).

Cuando se aplican los instrumentos lúdicos con niños de 4 y 5 años, se desarrolla el pensamiento, la memoria y la percepción mientras se divierte, es decir, se activan los procesos mentales mediante el juego con objetos concretos lo que humaniza al ser.

En la concepción vigotskiana, aparece como aspecto central, la noción sobre la zona de desarrollo próximo, en la cual, el autor se refiere a la diferencia entre el nivel de tareas que el niño y la niña puede realizar solo y las que puede llevar a cabo con ayuda de un mediador más competente, así la enseñanza que conduce a potenciar al niño, es la que está dirigida a la zona de desarrollo próximo, ello presupone la necesidad de la estructuración de niveles de ayuda que movilicen el pensamiento del niño y la niña, a través de la asimilación de nuevos aprendizajes.

Otro de los factores que intervienen en esta investigación sobre la aplicación de instrumentos lúdicos para el desarrollo de la motricidad fina es el movimiento, primera forma básica de comunicación humana con su entorno, que incluye, según Vigostky, el desarrollo motor en relación con lo histórico y cultural, en lo social, lo simbólico, lo volitivo, lo afectivo y lo intelectual, desde los primeros años de vida.

Así, la acción del mediador-a, es fundamental y una forma de aportar, es la de activar esta zona de desarrollo próximo, ¿cómo?, proponiendo instrumentos lúdicos que apasionen al infante y que su manipulación procure, no solo movimientos puntuales que favorezcan el desarrollo de la motricidad fina, sino que también le diviertan mientras aprende.

El niño disfruta de la lúdica; el juego le presenta desafíos para desarrollar los movimientos y esto debe traducirse en aprendizajes, una pedagogía para promo- 
ver el desarrollo motor, el desarrollo sensorial del niño y niña, la coordinación óculo manual, entre otros aspectos. Con la manipulación de los instrumentos lúdicos los infantes se divierten, en un ambiente de aprendizaje multidimensional como parte de su desarrollo integral que promueve el buen vivir expandiendo su felicidad.

\section{¿Qué son los instrumentos lúdicos?}

Considerados un componente pedagógico; un objeto lúdico es un juguete, pero no todo juguete es objeto lúdico, más en la actualidad donde hay juguetes que juegan solos y no generan nada en quien los posee o interactúa con ellos.

Los instrumentos lúdicos asociados al movimiento de los músculos finos, "son un conjunto de objetos concretos manipulables que aplicados adecuadamente y en forma lúdica, desarrollan en los niños determinada acción motriz fina, contribuye de manera muy efectiva al desarrollo de la habilidad digital, máxima expresión del desarrollo motor humano".

La finalidad de los instrumentos lúdicos es que genere movimiento, diversión, procesos mentales en las personas; de hecho, la finalidad primaria del juego es que los niños y niñas aprendan por intermediación de un objeto o acciones. En este caso, los objetos lúdicos propuestos por el proyecto, son tomados del entorno, construidos manualmente y de la experiencia de su aplicación, motivan y despiertan el interés del niño y la niña y su uso frecuente contribuirá al objetivo propuesto.

George Bernard Shaw (1925) plantea que los entornos lúdicos potencian el aprendizaje, al considerar que: "aprendemos el 20\% de lo que escuchamos, el 50\% de lo que vemos y el $80 \%$ de lo que hacemos. A través de entornos lúdicos en base a la metodología experiencial potenciamos al 80\% la capacidad intelectual".

Se propone que los niños y niñas manipulen el material concreto, proceso en el que interviene la experiencia lúdica, para un mayor aprendizaje.

La ludi expresión es el juego en acción, una manera de desarrollar la creatividad y compartir el conocimiento desde lo lúdico; esto es posible mientras el niño y la niña juega y desarrolla las habilidades de pensamiento. Es jugando donde el infante despierta los sentidos, explora sentimientos y pensamientos así como desarrolla la psicomotricidad, la expresión corporal, el movimiento y ritmo, la postura corporal, el sentido del espacio y tiempo; es en la práctica del juego con sus iguales donde piensa y se divierte, fortaleciendo su relación consigo mismo y la vinculación con los demás. Según Huizinga: "los animales juegan pero solo el hombre se divierte". Los infantes se divierten, gozan y aprenden, así los instrumentos lúdicos cumplen el objetivo.

Por medio del juego aprende de los demás y encuentra un sentido en sí mismo, fortalece su identidad, hereda la cultura y aprende de su entorno y del mundo que le rodea. El juego adoptado como estrategia en el nivel inicial desarrolla la creatividad, denominada también pensamiento original, pensamiento creativo, inventiva, imaginación constructiva o pensamiento divergente, es la capacidad de 
crear, de innovar, de generar nuevas ideas o conceptos, o nuevas asociaciones entre ideas y conceptos conocidos, que normalmente llevan a conclusiones nuevas, resuelven problemas y producen soluciones originales y valiosas.

\section{¿Y cuál es la diferencia con los materiales didácticos?}

Marquès, P. (2010) "Un recurso didáctico es cualquier material que maestros o alumnos elaboren, seleccionen y utilicen para apoyar los procesos de enseñanza y de aprendizaje".

Los materiales didácticos, también denominados auxiliares didácticos o medios didácticos, pueden ser cualquier tipo de dispositivo diseñado y elaborado con la intención de facilitar un proceso de enseñanza y aprendizaje, en tanto los instrumentos lúdicos permiten aprendizajes significativos a través de la diversión.

\section{¿Qué es la motricidad fina?}

Es toda acción que comprometa el uso de las partes finas del cuerpo: manos, pies, dedos, músculos finos de la cara. La destreza lograda de estas partes finas del cuerpo constituye el desarrollo de la motricidad fina, un elemento de esta destreza es la coordinación viso manual y otras habilidades como la dirección, precisión y puntería, movimientos de la pinza digital indispensables en aprendizajes futuros como la lectoescritura (Aguirre Zabaleta, 2001), sin perder de vista el proceso neurológico que se opera a partir del desarrollo motor grueso. La motricidad fina, constituye los movimientos finos en los que simultáneamente intervienen:

$$
\begin{aligned}
& \text { Ojo - mano } \\
& \text { Ojo - pie } \\
& \text { Ojo - mano - pie } \\
& \text { Dedos }
\end{aligned}
$$

El desarrollo de la motricidad fina empieza a tempranas edades (Bird 1998), a través del tiempo, de las experiencias, vivencias, referencias espacio - temporales, conocimiento, factores culturales, sociales, anatómicos, biológicos, entre otros, (Woodard y Surbug, 2001) un proceso que no termina nunca, sin embargo es hasta los 6 años, y así confirman los estudios al respecto, en que se debe estimular, en atención a la maduración neurológica y física.

La infancia es la "edad de oro" del aprendizaje motor, de la plasticidad del movimiento, convertido en un arte natural y espontáneo. Es un proceso complejo y exige la participación de muchas áreas corticales, hace referencia a la coordinación de las funciones neurológicas esqueléticas y musculares utilizadas para producir movimientos precisos.

Los avances científicos determinan los estudios del cerebro y la maduración neurológica para encontrar explicación a la importancia de esta capacidad humana que diferencia de los animales, pues son sus movimientos "dotados de sentido útil" expresiones de ideas y sentimientos posibles de ejecutarlos a través del movimiento motor grueso y motor fino, que llevan al infante a su independencia y a la realización de acciones más complejas cada vez, hasta lograr perfeccionarlas. 
Wallon Henri (1941) psicólogo y pedagogo francés ya dijo: “El movimiento es la única expresión de lo psíquico, concibe determinantes biológicos y culturales del desarrollo del niño y la maduración del sistema nervioso". Sus amplias concepciones al respecto, sustentan la importancia del desarrollo motor hasta lograr precisiones muy particulares como la que nos ocupa.

Por tanto, la motricidad fina es el refinamiento del control de la motricidad gruesa y se desarrolla a medida que el sistema neurológico madura; así, el dinamismo motor es el punto de partida de la construcción de la inteligencia y sus diversos factores.

Cobra reconocimiento como proceso de aprendizaje primordial para el desarrollo integral y no es difícil detectar en las primeras edades debilidades a nivel motor grueso como correr, saltar, girar, mantener el equilibrio en un solo pie, confusión en cuanto a la lateralidad y hasta un débil reconocimiento de su esquema corporal.

En el centro infantil son aún más notorias estas debilidades, niños y niñas que a sus 5, 6 años muestran dificultades para vestirse, atarse los cordones, manejarse en grupo, uso del lápiz, realización de sencillos trabajos como pintar, delinear, rasgar, entre otros, lo que impide el disfrute de sus acciones y limita el progreso y las aspiraciones de continuar adelante con nuevos retos. Surge así la minimización de sus capacidades, que coloca al niño en franca desventaja de otros niños y niñas que si han logrado el dominio de esta habilidad motora (Herrero, 2000).

A futuro, frente a nuevos aprendizajes como el proceso de lecto-escritura en el que interviene desde la postura corporal con un control adecuado, la orientación espacio temporal, coordinación motriz, organización viso manual, funciones ejecutivas como la atención, la memoria y desde luego la motricidad fina que exige capacidades motrices perceptivas, simbólicas y relacionales que van evolucionando conjuntamente con el desarrollo psicomotor, es muy probable que surjan las primeras dificultades de aprendizaje, la más significativa refiere a los procesos de lecto-escritura que anulan la formación de hábitos.

En 2013, en una encuesta del Instituto Nacional de Estadística y Censos (INEC), realizada en cinco ciudades del Ecuador, 27\% de las personas entrevistadas declaró no tener hábito de leer. De ellas, 56,8\% dijo que no le interesaba la lectura. Entre quienes dijeron leer libros, $0.3 \%$ dijo hacerlo en una biblioteca. ¡Qué daría una encuesta nacional que incluyera las ciudades pequeñas, las zonas rurales, la población menor de 16 años! (Rosa María Torres, exministra de Educación en Ecuador)

Con estas evidencias, la educación básica habrá perdido la posibilidad de formar no solo grandes lectores, sino también escritores, pintores, bailarines, músicos, entre otros.

Es así, como el triste comienzo se evidencia con las dificultades caligráficas muy comunes en nuestro contexto nacional y el tortuoso trabajo por mejorar, repetición de planas de escritura sin sentido que no logran ni el desarrollo tónico de la musculatura ni el objetivo propuesto. 
He aquí la importancia de esta investigación: determinar el desarrollo motor fino en una población representativa del país, a través de la aplicación de instrumentos lúdicos.

Para el efecto el grupo investigador se planteó 5 fases a desarrollar en este proyecto:

1. Búsquedas y sustento científico de las variables.

2. Alianzas estratégicas institucionales.

3. Fase de pilotaje.

4. Aplicación de instrumentos de investigación.

5. Hallazgos y logros de la investigación.

Las variables propuestas determinaron la población, se consideró la provincia de Pichincha, área urbana, como la población recomendable para la fase de pilotaje. Un primer proceso beneficioso que permitió revisar el instrumento de investigación, reconstruirlo y validarlo una y otra vez. De igual manera se seleccionaron los instrumentos lúdicos y se construyeron los más adecuados para este proceso investigativo.

\section{E1 Instrumento de investigación}

Se determinó el más idóneo, una lista de cotejo. ¿Por qué se determinó como instrumento de evaluación la lista de cotejo?. Simplemente porque el proceso de observación fue individual y con tiempos marcados lo que genera fácilmente información, además, la aplicación del Alfa de Crombach, con $0,80 \%$ confirma la validez de este instrumento de investigación.

La lista de cotejo está conformada con 10 ítems que miden el desarrollo motor fino, a partir de algunos elementos (Aguirre Zabaleta) como:

El biorritmo, es decir, el movimiento sincronizado que el niño realiza para enroscar la tuerca en el perno, al realizar el movimiento con sus dedos índice y pulgar hasta llegar a la cabeza del perno lo realiza en forma rítmica.

La coordinación óculo manual, al entregar al niño un espiral con 30 anillos y una cuenta pequeña iniciada la primera vuelta, hacerla girar alrededor de los anillos hasta terminar el espiral.

La coordinación viso motriz y eutonía muscular, se entregó a cada niño 30 tapas corona (tillos) y una pinza para sujetar ropa para que tome cada tapa y coloque en un recipiente.

Coordinación óculo motriz, pinza digital, euritmia y presión, el instrumento para determinar esta habilidad es la caña de pescar elaborada de un palo de pincho sostenido por una cinta y una cuenta, en un extremo adherido un imán de buena calidad para que el niño pueda pescar y colocar las tapas en un recipiente. Se observó que el niño pesque el mayor número de tillos utilizando la mano dominante y venciendo la dificultad de envolver y desenvolver la cinta en el palo de pinza. 
Habilidad digital, pinza digital, a través de un minicircuito construido con un clip mariposa y 30 canutillos de $5 \mathrm{~mm}$ para ensartarlos y llenar los tres lados.

La eutonia, se midió con el apoyo de una tabla multiuso y 18 parantes de sorbete grueso para que el niño plante en los orificios marcados haciendo presión. (Eutonia: tono corporal óptimo para el normal funcionamiento cinético y distonía al desajuste funcional de dicho tono, bien por exceso de tensión, hipertonía, o por déficit, hipotonía. Brieghel- Muller, 1994)

Coordinación óculo manual, prehensión y pinza digital, en los parantes debe insertar 1 cuenta y un palillo cada vez, 18 en total.

Se puede evidenciar que en la construcción de la lista de cotejo se tomaron en cuenta todos los aspectos que determinan el desarrollo de la motricidad fina.

Para llegar a la definición de esta lista de cotejo, se revisaron instrumentos estandarizados como el que presenta la Escala de Evaluación de la Psicomotricidad en preescolar (EPP) de la autoría de Ma. Victoria De la Cruz y Ma. Carmen Mazaira de procedencia española, ediciones TEA, para evaluar algunos aspectos de la psicomotricidad: locomoción, equilibrio, coordinación y conocimiento del esquema corporal. Para niños de 4 a 6 años, entre otros.

En el país nada se encontró para determinar motricidad fina, lo que llevó a la construcción de un instrumento propio a ser probado una y otra vez con diferentes grupos de niños, hasta lograr su construcción definitiva por parte del equipo investigador.

Se probaron instrumentos lúdicos así tambien se examinaron diferentes indicadores para la construcción definitiva del instrumento de investigación y aplicación en la fase de pilotaje, con una población de infantes de 615: 275 niños, 44.71\% de la población y 340 niñas, 55.28\% de la población que asisten al Instituto Superior Educativo "Consejo Provincial de Pichincha". Una experiencia única para iniciar el camino paso a paso del proceso investigativo.

\section{Cuáles son los resultados de esta fase de pilotaje}

El mayor logro de desarrollo de motricidad fina es alcanzado por las niñas en relación con el desarrollo de motricidad fina de los niños.

Qué explicación encuentra la investigación frente a estos datos, confrontados con la aplicación del instrumento dirigido a las educadoras.

\section{Cuáles son los hallazgos en esta fase de pilotaje}

1ero, en la ciudad los niños y las niñas están más en contacto con la tecnología, manejan fácilmente el celular de sus padres, sin que esto quiera decir que sea lo mejor,

2do, que las niñas desde muy pequeñas están más involucradas en las tareas del hogar, más que los niños. Ayudar a sus madres en los quehaceres domésticos, como desgranar, escoger, cortar, picar, entre otros, favorece el manejo de manos y dedos. 
3ero, las educadoras aseguran que los trabajos de las niñas, evidencian mayor prolijidad que los de los niños.

4to, las niñas demuestran mayor paciencia en la ejecución de sus trabajos.

Paralelamente a este proceso, con el aporte de Mariusz Wolonciej PhD del programa PROMETEO (Senescyt) integrado al proyecto, se consideró necesario diseñar un instrumento de investigación, cuestionario, para recoger información de 559 educadoras de los primeros años de educación básica, sobre la relación entre el desarrollo de la motricidad fina y los aprendizajes de lecto-escritura.

El 90\% de docentes investigadas, por el conocimiento y la experiencia vivida en el aula, están de acuerdo que la motricidad fina determina procesos de lectoescritura y su desarrollo se da en los primeros años de vida del niño y la niña.

En la búsqueda de investigaciones similares en otros lugares del planeta, se encuentran resultados con este criterio, lo que lleva a pensar que este es un primer hallazgo significativo de esta investigación, dejando de ser una suposición.

En un tercer momento, se seleccionó la población motivo de la investigación:

Provincia de Cotopaxi, cantones Pujilí y Saquisilí, Pujilí con su parroquia urbana Pujilí y dos parroquias rurales Guangaje y Zumbahua, con sus tres zonas geográficas donde se encontraron mestizos, montubios e indígenas un aprendizaje enriquecedor.

Manabí con una muestra representativa de la región Costa, ciudades de Manta y Portoviejo.

Provincia Francisco de Orellana, con su capital Francisco de Orellana, Cantones Loreto y Aguarico, una muestra interesante de esta región de la Patria.

Región Insular, Isla Santa Cruz, una evidencia de la diversidad de su población, constituida por colonos y asentamientos diversos de las regiones Costa, Sierra y Oriente.

El total la población infantil investigada es de 1963 niños y niñas de 4 y 5 años de edad que asisten a centros educativos fiscales. Una población que dobló el número previsto de infantes.

¿Cómo fue posible investigar a este número de niños y niñas?. Con el aporte de 180 estudiantes, 148 mujeres y dos varones de la carrera de Educación Parvularia, estudiantes de los 5tos y 6tos semestres que reciben la disciplina de Evaluación de Aprendizajes en su malla curricular, capacitadas y entrenadas por su maestra para participar en este proceso de investigación y la participación de un paralelo de primer semestre, capacitado por su profesor, para cumplir estos objetivos.

\section{Hallazgos de la investigación}

Cada lugar investigado arroja información valiosa sistematizada en los resultados finales del proyecto como producto esperado de la investigación.

En una descripción de la experiencia en la provincia de Cotopaxi, ubicada en la serranía ecuatoriana, se seleccionaron instituciones del nivel inicial 
ubicadas en la ciudad de Pujilí ubicada a una altitud de $2961 \mathrm{msnm}$. a $10 \mathrm{~km}$. al Occidente de Latacunga. Se tomó la cabecera cantonal Pujilí, área urbana y 2 parroquias rurales Zumbahua y Guangaje del área rural, entre ellas la Unidad Educativa del Milenio "Cacique Tumbalá"

Zumbahua, un contexto riquísimo con resultados de interés investigativo, una parroquia rural donde se evidencia pobreza y desnutrición. Un gélido clima que mantiene resfriados a los niños más pequeños, prueba de ello, sus narices fluyendo constantemente, rostros oscuros y deshidratados, manos entumecidas y torpes para un trabajo que implica movimiento fino, este y otros hechos son motivo de investigación.

Los niños visten el uniforme que el Estado les proporciona, el desayuno escolar a media mañana, para muchos de ellos es su primera comida.

Ante la visita y propuesta del trabajo sus rostros se iluminaron y ansiosos esperaron su turno, la mayoría silenciosos y tímidos lleva a pensar, qué importante es continuar fortaleciendo su desarrollo integral y personalidad, así como trabajar con sus familias y comunidad.

Las instituciones investigadas en el cantón Pujilí resumen lo siguiente:

374 infantes, 178 niñas $47.5 \%$ y 196 niños, 52,4\% niños, se evidencia un leve mayor logro de motricidad fina en niñas en relación a los niños.

Qué explicación arroja la investigación, cuál es el hallazgo significativo:

1ero Las niñas en esta región del país están más ligadas a las actividades de la madre, desde muy pequeñas ayudan a desgranar, a seleccionar semillas y a introducirse en el manejo de herramientas para el tejido de shigras.

2do La concepción machista no permite que el niño realice tareas del hogar, para él está el juego y para la niña el trabajo de hogar.

3ero A criterio de las educadoras, es mayoritario el número de niñas que realiza trabajos en el aula, con mejores acabados, prolijidad y dedicación. Se evidencia una relación directa entre el desarrollo de la motricidad fina y los aprendizajes.

Saquisilí. El cantón alcanza una altitud que varía entre 2900 y 4200 msnm. Una población con el 65\% indígena y 35\% mestiza. La pobreza alcanza el 84.16\% de la población total del cantón (INEC 2010).

La mayor población es joven, analfabetismo en mujeres $29,16 \%$ en varones el $13.84 \%$. Una ciudad muy particular seleccionada para la investigación, sus páramos extensos acogen a muchos niños y niñas y en su búsqueda llegó el equipo investigador.

La aplicación del instrumento registra lo siguiente: incremento del desarrollo de la motricidad fina en niños más que en niñas.

\section{Qué explicación da la investigación:}

Los niños tienen mayor libertad para el juego a campo abierto y su juego favorito consiste en hacer bailar con el manejo de la pinza digital a la semilla del 
eucalipto al que le denominan "el baile de chuspi", algo similar como "el baile del trompo". Un juego al que se integra grupos de niños desde tempranas edades y organizan competencias entre ellos.

Este juego no solo ha desarrollado la motricidad fina, se constituye en el camino a la matemática, los niños saben hacer cuentas y conocen cuántas veces van ganando y cuántas veces van perdiendo. Son datos que fortalecen las variables de la investigación.

Qué pasa con las niñas, su condición les limita desde pequeñas a ejercer el derecho a jugar, a actuar y a desarrollarse como tales.

La investigación realizada en Manabí resume estadísticas interesantes que dan los siguientes datos:

Población investigada 252 infantes: 123 niñas, 48\%. 129 niños, 51,1\% de la población.

Los datos revelan que en los niños la motricidad fina está mejor desarrollada en relación con las niñas, encontrándose una diferencia significativa.

Hallazgos de la investigación: la relación del niño en el entorno de libertad el que crece y sobre todo la incorporación a las tareas de su padre, en el caso de los niños de Manta que se encuentran muy vecinos a la playa, evidencia el acercamiento al manejo de herramientas para la construcción de redes de pescar en la mayor parte de los casos y la posibilidad de juegos en el patio de sus casas y centros educativos. Ejemplo el juego de tillos y canicas para ser sacadas del círculo que dibujan en el piso.

Esto, se comprobó al mirar sus bolsillos con canicas y otros objetos que utilizan con el mismo fin.

Algunos niños que no disponen de canicas, se dan modos para jugar a lo que les gusta y se han ideado un juego de coordinación viso motriz. Realizan un pequeño orificio en la tierra y en la arena, lugar donde colocan una piedra pequeña y luego lanzan otra de forma plana hasta sacarla. El niño que logra sacar varias veces la piedra, gana el juego.

Gana el más diestro, a este juego se le conoce como "Ilieny". En el aula se observó que por la infraestructura de la mayor parte de las instituciones, están juntos niños y niñas y las educadoras proponen trabajos homogéneos en los que se ayudan mutuamente, lo que permite concluir que en el aula niños y niñas tienen las mismas oportunidades para desarrollar estas habilidades, sin embargo, se determina que son otros factores del contexto los que marcan las diferencias individuales.

Un detalle interesante, en los niños de la provincia costera: su actitud más abierta y segura les lleva al disfrute de cada actividad de la investigación propuesta, sobre todo en la pesca de objetos, en contraste con el gesto tímido y esquivo de los niños del páramo andino.

La provincia Francisco de Orellana, la capital que lleva el mismo nombre, el cantón Loreto y Aguarico fueron considerados para esta investigación, como una muestra de la región amazónica. 
En la ciudad de Orellana y en el cantón Loreto, los datos evidencian una ligera diferencia en el logro de la motricidad fina en niños en relación a las niñas.

Sin embargo, se encontró que una actividad propia de la región, es la elaboración de artesanías, tejido de cestas elaboración de collares, pulseras en base a semillas, actividad en la que se involucran más las niñas que los niños.

Un tema pendiente a profundizar en la investigación puede ser la particularidad de esta "región petrolera" que empieza cambiando la vida de sus pobladores, por solo este hecho.

Prueba de ello es que existe una matrícula de niños de 4 y 5 años de más de 2 000 infantes, ubicados en los centros infantiles. La tasa de natalidad en estos últimos años, se ha elevado significativamente (INEC 2010).

Niños y niñas con una realidad muy particular, tanto el niño como la niña, están en iguales condiciones de buscar su propia atención, como por ejemplo vestirse solos desde muy temprana edad, buscar sus alimentos e incluso responsabilizarse de sus hermanos más pequeños.

Un gran porcentaje de madres están muy ocupadas en sus propias actividades en tanto los padres están ausentes de sus hogares por situaciones de trabajo, lo que altera el normal crecimiento, desarrollo y madurez de los niños y niñas y esto se refleja en sus comportamientos.

En los centros educativos las educadoras han dado valor al desarrollo de la motricidad fina, mediante la práctica del tejido en esterilla. Fue común observar trabajos como portarretratos, adornos, cestas, elaboradas prolijamente por los niños y niñas.

Esta gran población infantil imposible de ser tomada en cuenta en su totalidad, dejó muchas expectativas y caminos abiertos para la investigación.

En esta vez se dispuso de un día con la colaboración del equipo investigador conformado por 96 personas. Sin embargo se considera un logro el haber aplicado el instrumento de investigación a 477 niños y niñas distribuidos así: 247 niños, $51.7 \%$ de la población; 230 niñas, 48.2\% de la población.

En el cantón Aguarico al que el equipo investigador llegó después de un día por vía fluvial, se encontró con una institución educativa en Nuevo Rocafuerte con una población de origen quechua y wuahorani, un grupo de niños y niñas trilingües, que hablan el idioma castellano, quechua y wao.

Las viviendas construidas de madera de monte, adentradas en la selva, están dispersas en toda zona, los niños y niñas caminan largas distancias hasta ser recogidos por una canoa del gobierno local para ser llevados hasta el centro infantil.

Las condiciones en las que se desenvuelve esta población infantil son difíciles y determinantes para su proceso de educación formal. Las lluvias permanentes impiden la movilidad adecuada y regular para llegar a la institución; por esta razón la asistencia no es obligatoria y no existe un proceso de recuperación pedagógica, por lo que su nivel educativo es limitado. 
Por otro lado, la mayoría de docentes no son profesionales de la educación, esto en el nivel inicial; asumiendo que su tarea se convierte en cuidado y atención a los infantes, minimizando el proceso educativo.

18 infantes: 7 niñas, 38 . \%, y 11 niños, 61.4\% de la población.

Hallazgos de la investigación: se evidencia mayor desarrollo de la motricidad fina en niños que en niñas.

Tiputini, ubicada en el límite con Perú, una población de 19 infantes: 15 niñas, $78.9 \%$ de la población, y 4 niños, $21 \%$ de la población.

Hallazgos: en esta población de acuerdo con los resultados estadísticos: en los niños se evidencia muy poca diferencia, en el desarrollo de la motricidad fina con relación a las niñas, ya que en estas edades no es significativa la diferencia de género en relación con la motricidad fina. La investigación responde de la siguiente manera:

Las niñas se involucran muy temprano a la manipulación de semillas como los "guaruros" propias de la región para la elaboración de artesanías, pulseras y collares.

Los niños desde tempranas edades acompañan a sus padres en labores de campo, caza y pesca. Cuando crecen son tan hábiles para esto último que no es nada extraño mirarles en las orillas de los ríos con una caña de pescar, disfrutando de los logros y conquistas en este proceso de desarrollo.

Región Insular, Isla Santa Cruz, total de la población investigada 90 infantes: 41 niñas $45.5 \%$; 45 niños, $50 \%$ de la población.

Las estadísticas evidencian igual desarrollo de la motricidad fina en niños y niñas; sin diferencias de género. La investigación explica igualdad de oportunidades para el logro de la habilidad; en estas edades tempranas no existe divergencia de género. El resultado de la investigación se confirma con otras investigaciones en similares contextos (E Ferreiro, A. Teberosky, 1991). Durante la investigación se comprobó que las instituciones educativas fortalecen el desarrollo de la motricidad fina en un ambiente lúdico y de seguridad.

La isla Santa Cruz registra una población de colonos de la parte central de la Sierra. Llama la atención un grupo representativo de comunidad de Cañar que han logrado del gobierno seccional, la creación de una escuela bilingüe con énfasis en la enseñanza del idioma quichua y respeto a su identidad cultural.

Así, expuesto el resultado de esta investigación, conviene añadir algunas conclusiones producto de la sistematización de la aplicación de los instrumentos de investigación a padres y educadores del nivel inicial.

1. El desarrollo de la motricidad fina responde a un proceso de maduración neurológica desde tempranas edades y son varios los factores determinantes, biológicos, psicológicos, esqueléticos, musculares utilizados para producir movimientos precisos. La neurociencia explica ampliamente el proceso que parte del desarrollo de la motricidad gruesa para llegar a la motricidad fina. 
2. Es producto cultural, el estudio revela que no existen diferencias significativas de género entre región y región, los niños y niñas de las provincias investigadas encuentran en su entorno herramientas para el desarrollo del niño y la niña, y en este caso muy específico para el desarrollo de la motricidad fina. El estudio llegó a esta conclusión, revisando aportes como el de Marvin Harris, (antropólogo norteamericano de renombre, 2001) la Cultura es "el conjunto de tradiciones y estilos de vida socialmente adquiridos de los miembros de una sociedad incluyendo sus modos pautados y repetitivos de pensar, sentir y actuar, es decir su conducta”.

El autor encuentra explicación a tantos comportamientos humanos ya sea en su relación entre los miembros de la sociedad la más pequeña, como es la familia y la relación de sus miembros con otros entornos sociales. De la misma manera las diferentes ciencias desde su especialidad tratan de explicarse la esencia del hombre, su mente y su alma.

La medicina, la psicología, sociología, la antropología, la geografía humana, la psicología social y aquí su mayor representante Geert Hofstede (1980) y sus cuatro dimensiones, la historia, las ciencias políticas, la economía, la lingüística, la teología, la filosofía, la museología, el arte, la literatura. La antropología como tal, va más allá de este estudio parcializado del comportamiento humano, cuando hace uso de sus investigaciones y conclusiones y las somete a un estudio correlacional entre comunidades, grupos multiétnicos para determinar patrones referenciales.

Es así como Harris determina para el estudio de la cultura, patrones de la cultura universal, en tres dimensiones: estructura, infraestructura y supraestructura. Los aportes de este antropólogo son valorados por la humanidad, pues simplifican el conocimiento de la cultura, a partir de compresión de la necesidad de definir por ejemplo,

- una estructura del conocimiento social,

- la transmisión de conocimientos,

- la relación del hombre con el ambiente, entre otros.

Aspectos relacionados con la subsistencia del hombre en el ambiente, la reproducción, la organización del intercambio de bienes y trabajo, la vida en el seno de grupos domésticos y comunidades más amplias, así como los aspectos creativos, expresivos, lúdicos, estéticos, morales e intelectuales de la vida humana.

Parámetros todos estos, que bien se pueden tomar en cuenta para efectos de la investigación de procesos de aplicación de instrumentos lúdicos para el desarrollo de la motricidad fina en niños y niñas de 4 a 5 años, considerando variables de género, etnicidad, clase y territorial, incluye uso de TIC. Investigación que se propuso en su cuarta fase, comprender los patrones culturales de las diferentes regiones donde se aplicó el instrumento, para determinar motricidad fina, así: prueba piloto, Unidad Educativa Consejo Provincial de Pichincha, (Quito); Cotopaxi, Pujilí, Saquisilí; Manabí, Manta, Portoviejo; Francisco 
de Orellana, Loreto, Coca; Región Insular, Isla Santa Cruz, con los resultados ya descritos.

La educación debe aprovechar y valorar el contexto en el que se desarrolla el niño y la niña para maximizar sus capacidades, entre ellas la motricidad fina, tema que nos ocupa.

3. El desarrollo motor es determinante en la vida de los seres humanos y sobre todo de los niños y niñas que empiezan a los 4 y 5 años un proceso de aprendizaje hacia el desarrollo de habilidades cognitivas y sociales.

4. Los niños y niñas necesitan espacios para el movimiento, la actividad lúdica es fundamental para su desarrollo.

5. El juego inspira la iniciativa y creatividad en ese compartir con sus iguales.

6. Las instituciones educativas deben tomar en cuenta las características del desarrollo infantil y procurar oportunidades para favorecer procesos para la consecución de logros en estas edades.

7. En este proceso investigativo se resalta la frase "nada tiene valor si no está de por medio el corazón” y es esta la vivencia que ha elevado la sensibilidad de los investigadores y del grupo de colaboradores, quienes han tenido la oportunidad de involucrarse.

\section{Bibliografía}

Aguirre Zabaleta, J. (1996). Desarrollo de la inteligencia motriz, como base para la ejecución y capacitación. España.

Arnaiz, P. (1987). Evolución y contexto de la práctica psicomotriz. Murcia, España.

Arnaiz, P. (1999). Psicomotricidad y educación. Madrid, España.

Asamblea Nacional Constituyente (2008). Constitución de la República del Ecuador.

Berruezo, P.P. (2000). El contenido de la psicomotricidad. Madrid, España.

Bird, A. (1998). Enseñando educación física. Carolina. Puerto Rico: Logo.

Bloom, B. (1967). Importancia e influencia del primer medio ambiente y la primera experiencia. Madrid.

Harrison, M. (2000). Teorías sobre la cultura en la época postmoderna. Primera edición en español. EE.UU.

Lázaro, A. (1995). Radiografía del juego en el marco escolar propuesto. En Psicomotricidad, revista de estudios y experiencias. España.

Montessori, M. (1987). El niño y el secreto de la infancia. México.

Otsky, L.S. (1982). El juego y su función en el desarrollo psíquico del niño. Revista Cuadernos de Pedagogía, nro. 85, España. 
Rigail, R. (2006). Educación motrizy educación psicomotriz en prescolar y primaria. Barcelona, España.

Senplades (2013-2017). Plan Nacional para el Buen Vivir

Sugrañes, E. y Angel, M.A. (2007). La educación psicomotriz. (3-8 años). Cuerpo, movimiento, percepción, afectividad: una propuesta teórico-práctica. Barcelona.

Taggart, A., Keegan, A. (1997). Developing fundamental movement skills in oudoor settings.

Vygotsky, L. S. (1978). Mind in society. Cambridge, MA: Harvard University Press.

Wallon, H. (1941). La evolución psicológica del niño. Barcelona.

Watson Brown, Herminia et al. (2008). Teoría y práctica de los juegos. La Habana, Cuba: Editorial Deportes. 